\title{
Estrategias de afrontamiento, estrés percibido $y$ bienestar psicológico en individuos con cefalea primaria
}

\author{
Felipe E. García; María Alejandra Catrilef; Yohanna Fuentes; Sandra Garabito; Valentina Aravena
}

Cómo citar este artículo:

García, F. E., Catrilef, M. A., Fuentes, Y., Garabito, S., \& Aravena, V. (2021). Estrategias de afrontamiento, estrés percibido y bienestar psicológico en individuos con cefalea primaria. Acta Colombiana de Psicología, 24(1), 8-18. https:// www.doi.org/10.14718/ACP.2021.24.1.2

Recibido, enero 12/2019; Concepto de evaluación, agosto 21/2020; Aceptado, agosto 24/2020

Felipe E. García ${ }^{1}$

ORCID: https://orcid.org/0000-0002-4161-5840

Universidad Santo Tomás, Concepción, Chile.

Universidad de Concepción, Concepción, Chile.

María Alejandra Catrilef

ORCID: https://orcid.org/0000-0003-1871-9825

Universidad de Las Américas, Concepción, Chile.

Yohanna Fuentes

ORCID: https://orcid.org/0000-0002-2133-3934

Universidad de Las Américas, Concepción, Chile.

Sandra Garabito

ORCID: https://orcid.org/0000-0002-3152-5067

Universidad Santo Tomás, Concepción, Chile.

Valentina Aravena

ORCID: https://orcid.org/0000-0002-0160-5859

Universidad de Concepción, Concepción, Chile.

Resumen

\begin{abstract}
El propósito de este estudio fue evaluar la asociación entre las estrategias de afrontamiento, el bienestar subjetivo y el estrés en personas con cefalea primaria —es decir, con dolor de cabeza sin origen orgánico—. En total, participaron 150 personas adultas - 135 mujeres y 15 hombres_- diagnosticadas con cefalea primaria y episodios de cefalea en los últimos seis meses. Los instrumentos de medida utilizados fueron la Escala de satisfacción con la vida de Diener, la escala de estrategias de afrontamiento Brief Cope, la Escala de estrés percibido de Cohen, dos preguntas sobre la intensidad y frecuencia del dolor, y un cuestionario sociodemográfico. En general, se encontró que el bienestar subjetivo se relaciona positivamente con la planificación y negativamente con el uso de sustancias y la frecuencia del dolor; a la vez que el estrés percibido se asocia positivamente con negar la realidad del acontecimiento estresante, culpabilizarse por la situación ocurrida y la frecuencia del dolor, y negativamente con la aceptación de la situación estresante. Además, se observó un mayor estrés percibido en usuarios de atención pública de salud que en aquellos que son atendidos en el sistema privado. Este estudio permite identificar las estrategias que deben ser potenciadas o modificadas con ayuda del personal de salud para mejorar la calidad de vida de las personas con cefalea primaria.

Palabras clave: calidad de vida, dolor de cabeza, evento estresante, satisfacción con la vida.
\end{abstract}

\footnotetext{
1 Facultad de Ciencias Sociales y Comunicaciones, Universidad Santo Tomás, avda. Arturo Prat 855, Concepción, Chile. C. P.: 4030000. Departamento de Psiquiatría y Salud Mental, Facultad de Medicina, Universidad de Concepción, Barrio Universitario s/n, Concepción, Chile. C.P. 4030000. Correo electrónico: fgarciam@udec.cl
} 


\title{
Coping strategies, perceived stress and psychological well-being in individuals with primary beadache
}

\begin{abstract}
The purpose of this study was to assess the association between coping strategies, subjective well-being and stress in people with primary headache, i.e., headache with no organically -based origin. In total, 150 adult persons participated -135 women and 15 men -diagnosed with primary headache and headache episodes in the last six months. The measurement instruments used were the Diener Life Satisfaction Scale, the Brief Cope Coping Strategies Scale, the Cohen Perceived Stress Scale, two questions on pain intensity and frequency, and a sociodemographic questionnaire. In general, it was found that subjective well-being is positively related to planning and negatively to substance use and frequency of pain; while perceived stress is positively associated with denying the reality of the stressful event, blaming oneself for the situation that occurred and the frequency of pain, and negatively with accepting the stressful situation. Also, greater perceived stress was observed in users of public health care than in those who are seen in the private system. This study allows the identification of strategies that should be enhanced or modified with the help of health personnel to improve the quality of life of people with primary headache.

Keywords: quality of life, headache, stressful event, satisfaction with life.
\end{abstract}

\section{Introducción}

La cefalea o dolor de cabeza es un problema de salud que representa un motivo de consulta frecuente en los servicios de salud. Según Hofstra et al. (2015), un $80 \%$ de la población ha reportado este problema en algún momento de su vida, $\mathrm{y}$, de acuerdo con la Organización Mundial de la Salud (oms, 2011), en la actualidad entre el $50 \%$ y el $75 \%$ de la población adulta lo padece, independientemente del sexo, raza, edad, niveles de ingreso o zonas geográficas. De hecho, la cefalea ha sido clasificada como la tercera causa de discapacidad a nivel mundial en hombres y mujeres menores de 50 años (Vos et al., 2016), y en Chile se estima que los dolores de cabeza se encuentran en el número uno de los problemas de salud que aquejan a la población (Ministerio de Salud, 2006). Asimismo, se calcula que la prevalencia mundial de cefalea en los adultos es aproximadamente del $50 \%$ (oms, 2016), aunque se reporta que solo un número reducido de ellos solicita atención medica por este motivo, lo cual ha dificultado identificar cabalmente los factores que caracterizan este trastorno (Waldie et al., 2015).

Sabiendo de este problema, la International Headache Society (IHS, 2018) divide las cefaleas en dos grandes categorías: (a) las cefaleas primarias, descritas como un dolor de cabeza intenso, con distintas duraciones, que se repiten cada cierto tiempo, el $99 \%$ no asociadas a una disfunción orgánica, y que incluyen la migraña, la cefalea tensional y las cefaleas autonómicas trigeminales; y (b) las cefaleas secundarias, que tienen un origen patógeno, ya sea por traumatismo encéfalo-craneano, abuso de sustancias, desórdenes vasculares craneales, infecciones, desórdenes psiquiátricos, entre otros, y que suman al dolor de cabeza otros síntomas asociados a la enfermedad orgánica de base.

Como es sabido, las cefaleas suelen ser recurrentes incluso crónicas - y afectan la calidad de vida de quienes las padecen (IHS, 2018), por ejemplo, al alterar sus ciclos de sueño (Benito-González et al., 2018) o al generar estrés en la familia (Operto et al., 2018). De hecho, respecto a esto último, se ha identificado que las cefaleas primarias parecen estar influidas por el estrés (OMS, 2006; Pascual, 2019; Schramm et al., 2014), y que estas, a su vez, generan un mayor estrés debido al mismo dolor al que está asociada; lo que ocasiona un círculo vicioso de estrés, dolor de cabeza y estrés (Martin, 2016).

Dentro de las cefaleas primarias, la de origen tensional es la más prevalente (oMs, 2006; Pascual, 2019), lo que se suma a que tiene una asociación importante con la aparición de síntomas depresivos y ansiosos (Tomé-Pires et al., 2016). Ahora, si bien por lo general las personas que padecen este problema reciben tratamientos farmacológicos, dicho tratamiento parece ser insuficiente (Dowson, 2003), lo que hace necesario que se complemente con atención psicológica orientada al reconocimiento de recursos y estrategias que posibiliten un mejor afrontamiento ante situaciones que provoquen estrés (Fumal \& Schoenen, 2008; Tomé-Pires et al., 2016), pues se ha encontrado que los factores psicológicos tienen un rol importante como contribuyentes tanto para la manifestación del dolor como para su mantenimiento (Operto et al., 2018).

Ahora bien, para brindar atención oportuna a pacientes con cefalea primaria es indispensable entender el concepto de dolor, el cual se ha definido, por una parte, como una 
experiencia sensorial y emocional desagradable asociada con daño tisular real o potencial (International Association for the Study of Pain, 1979); y, por otra —una definición más reciente-, como una experiencia somática mutuamente reconocible que refleja la aprehensión de una persona de la amenaza a su integridad corporal o existencial (Cohen et al., 2018). Además, se ha dicho que la experiencia del dolor es subjetiva y compleja, que no es ajena a ningún ser humano, que influyen en ella factores biológicos, psicológicos y sociales que se interrelacionan (Ramírez, 2002), y que el dolor a menudo constituye un problema complejo para quienes lo padecen, sumado a que es una de las causas más comunes por la que los pacientes acuden a consultas médicas (Del Monaco, 2013). De hecho, un tipo particular de dolor que es bastante común en las consultas es el orofacial, que afecta la cara y que suele estar acompañado de malestar emocional e interferir con las actividades de la vida diaria (oms, 2019).

Comprendiendo la dimensión del dolor, la afectación funcional y la influencia que el estrés tiene en este tipo de trastorno, resulta necesario establecer estrategias adecuadas para afrontar sus consecuencias. Al respecto, Lazarus y Folkman
(1984) plantean que el afrontamiento es una preparación para la acción que moviliza al individuo para evitar los daños del estresor, y que las estrategias de afrontamiento son un conjunto de esfuerzos tanto cognitivos como comportamentales relativamente cambiantes que se despliegan para manejar las demandas externas e internas, incluso cuando los sujetos se ven superados en sus propios recursos.

Según estos autores, cada individuo tiende a utilizar diferentes estrategias de afrontamiento dependiendo de los esfuerzos que cada uno realice y la forma en que equilibre sus reacciones emocionales. De hecho, se ha dicho que se adquieren por aprendizaje o por hallazgo fortuito en una situación de emergencia, y que pueden ser adaptativas -es decir, que reducen el estrés y contribuyen a la salud a largo plazo - o desadaptativas - aquellas que mitigan el estrés a corto plazo, pero no promueven la salud a largo plazo(Girdano \& Everly, 1986). Específicamente, Carver (1997) distingue catorce estrategias de afrontamiento, cuyas definiciones se pueden encontrar en la Tabla 1.

Ahora bien, en un estudio, Moretti y Medrano (2014) encontraron que las personas que tienden a quejarse de su dolor y que desarrollan conductas evitativas tienden a percibir

Tabla 1.

Estrategias de afrontamiento

\begin{tabular}{|c|c|c|}
\hline No. & Estrategia & Definición \\
\hline 1 & Afrontamiento activo & $\begin{array}{l}\text { Realizar acciones directas que permitan aumentar los propios esfuerzos de los individuos, para } \\
\text { reducir los estresores ambientales o eliminarlos definitivamente. }\end{array}$ \\
\hline 2 & Planificación & $\begin{array}{l}\text { Pensar en cómo enfrentar las diversas demandas, con el objetivo de planificar posibles accio- } \\
\text { nes que permitan direccionar los esfuerzos a realizar. }\end{array}$ \\
\hline 3 & Apoyo instrumental & $\begin{array}{l}\text { Pedir ayuda, consejos o información a personas especializadas respecto a la problemática } \\
\text { enfrentada. }\end{array}$ \\
\hline 4 & Uso de apoyo emocional & $\begin{array}{l}\text { Buscar apoyo emocional en personas que presenten características como simpatía } \\
\text { y comprensión. }\end{array}$ \\
\hline 5 & Autodistracción & $\begin{array}{l}\text { Capacidad de poder concentrarse en otros proyectos o en otras actividades con el fin de conse- } \\
\text { guir concentrarse en otros asuntos y no en el estresor. }\end{array}$ \\
\hline 6 & Desahogo & $\begin{array}{l}\text { Capacidad de reflexionar en el propio malestar emocional, junto a la tendencia de expresar o } \\
\text { canalizar esos sentimientos. }\end{array}$ \\
\hline 7 & Desconexión conductual & $\begin{array}{l}\text { Aminorar los esfuerzos para enfrentar al estresor, llegando incluso a desistir de los esfuerzos } \\
\text { para lograr las metas con las cuales interfiere. }\end{array}$ \\
\hline 8 & Reinterpretación positiva & $\begin{array}{l}\text { Capacidad de visualizar el lado positivo y favorable del problema, llegando a intentar solu- } \\
\text { ciones e, incluso, extraer aprendizajes de lo vivido, pudiendo llegar a alcanzar un crecimiento } \\
\text { personal. }\end{array}$ \\
\hline 9 & Negación & Negar la realidad del acontecimiento estresante. \\
\hline 10 & Aceptación & Admitir que lo que está ocurriendo es real. \\
\hline 11 & Religión & $\begin{array}{l}\text { Tendencia a refugiarse en la religión en situaciones estresantes, junto con el aumento en la } \\
\text { participación en actividades religiosas. }\end{array}$ \\
\hline 12 & Uso de sustancias & $\begin{array}{l}\text { Propensión a refugiarse en el consumo de alcohol u otras sustancias con el propósito de sen- } \\
\text { tirse mejor ante la situación que genera estrés. }\end{array}$ \\
\hline 13 & Humor & $\begin{array}{l}\text { Capacidad de reír o de hacer bromas a pesar de las situaciones estresantes, incluso haciendo } \\
\text { burlas de las mismas. }\end{array}$ \\
\hline 14 & Autoinculpación & Reprocharse y culpabilizarse por la situación ocurrida. \\
\hline
\end{tabular}

Nota. Adaptación a partir de Carver (1997). 
el dolor de forma más intensa, además de que las mujeres se quejan más verbalmente que los hombres, mientras que los hombres expresan mayor cantidad de quejas no verbales y desarrollan más conductas evitativas que las mujeres. Asimismo, Najam y Aslam (2010) encontraron que las personas que sufren de cefalea tensional y migraña tienden a utilizar estrategias de afrontamiento menos efectivas al momento de enfrentarse a situaciones de estrés, pues, en específico, aumentan las estrategias evitativas y disminuyen el uso de afrontamiento activo, además de que perciben las situaciones de estrés de forma más intensa.

Por otra parte, también se ha encontrado que la presencia de cefaleas primarias podría afectar el bienestar subjetivo de los usuarios afectados, siendo el bienestar subjetivo la autovaloración sobre qué tan valiosa es la propia vida —en relación con el concepto de satisfacción con la vida(Diener et al., 2003); aunque, según Torres y Flores (2018), no parece ser la salud objetiva en sí misma la que determina los efectos sobre el bienestar, sino, más bien, la percepción que se tenga sobre ella.

Ahora, respecto a la relación entre las estrategias de afrontamiento, el estrés y el bienestar, se ha encontrado que el uso de estrategias relacionadas con la búsqueda de apoyo social se vinculan con mayores niveles de satisfacción, y que quienes utilizan estrategias de resolución de problemas manifiestan mayor bienestar subjetivo que quienes usan sustancias o evaden las fuentes de estrés (Paris \& Omar, 2009). En particular, en un estudio con población chilena se observó que las estrategias de búsqueda de apoyo instrumental, afrontamiento centrado en el problema, aceptación y reinterpretación positiva se relacionan positivamente con el bienestar subjetivo, mientras que las estrategias de negación, autoinculpación, desconexión conductual, autodistracción y uso de sustancias se relacionan positivamente con el estrés percibido (Garcia et al., 2018; García et al., 2016).

Asimismo, en Chile, existen diferencias importantes respecto a la calidad del servicio entregado entre las prestaciones de salud pública y privada (Silva, 2018). Esto se traduce, por ejemplo, en que en la atención pública la comunicación con el usuario es menos expedita, los tiempos de espera son mayores, y el tiempo de atención por parte de los profesionales es menor debido a la mayor afluencia de público (Riveros \& Berné, 2007). Por lo demás, la mayoría de los pacientes que se atienden en el sistema público, por lo general, presentan menores ingresos económicos, y aunque las personas con mayores recursos no están ajenas a padecimientos como la cefalea primaria, la situación económica les brinda mayores posibilidades de generar actividades para reducir sus niveles de estrés —como realizar salidas, viajes, compras, etc.—.
Debido a todos los factores mencionados, y a la importancia de la cefalea como indicador de estrés en la vida moderna, el presente estudio buscó evaluar la asociación entre las estrategias de afrontamiento, el bienestar subjetivo y el estrés en personas con cefalea primaria. Además, se evaluó la influencia de la intensidad y la frecuencia del dolor percibido sobre el bienestar y el estrés, y como análisis complementario se hizo una comparación entre las personas atendidas en el sistema de salud público y las atendidas en el sistema de salud privado.

\section{Método}

\section{Diseño y tipo de estudio}

Se utilizó un diseño descriptivo y correlacional, y los datos fueron tomados en un solo corte temporal, lo que corresponde a un estudio transversal (Hernández et al., 2003).

\section{Participantes}

Se contó con un total de 150 participantes - $135 \mathrm{mu}-$ jeres $(90 \%)$ y 15 hombres (10\%)- de la provincia de Concepción, Chile; el promedio de edad correspondió a los 44 años $(D E=15.93$, rango de 18 a 86 años); y, del total de los participantes, el $69 \%(n=103)$ presentaba cefalea tensional, el $29 \%(n=43)$, migraña, y solo el $1 \%(n=2)$, cefalea en brotes. Como criterio de inclusión se consideró que los participantes hubieran padecido de algún tipo de episodio de cefalea en los últimos seis meses. Del total de personas participantes, el $58 \%(n=89)$ contaba con educación superior completa o incompleta.

\section{Instrumentos}

Los instrumentos de medida utilizados fueron la escala de estrategias de afrontamiento Brief Cope, de Carver (1997), la Escala de satisfacción con la vida, de Diener (1984), la Escala de estrés percibido, de Cohen (1983), dos preguntas sobre la intensidad y frecuencia del dolor, y un cuestionario sociodemográfico elaborado por los autores del estudio.

\section{Brief Cope}

Se utilizó la escala de estrategias de afrontamiento Brief Cope, elaborada por Carver (1997), en su versión validada en población chilena por Garcia et al. (2018), que consta de 28 ítems y 14 subescalas —afrontamiento activo, planificación, apoyo instrumental, uso de apoyo emocional, autodistracción, desahogo, desconexión conductual, reinterpretación positiva, negación, aceptación, religión, uso de sustancias, humor y autoinculpación-, 
con dos ítems cada una. Específicamente, esta prueba se responde en una escala tipo Likert de cuatro alternativas de respuesta —donde 0 corresponde a "nunca hago esto" y 3 a "hago siempre esto"-.

Esta escala es una versión más corta del inventario COPE (Carver et al., 1989), y fue diseñada especialmente para la investigación con personas aquejadas por problemas de salud, por lo cual se minimiza el tiempo dedicado a responder. En su propuesta original se obtuvieron los 14 factores mediante una análisis factorial exploratorio, y en su validación chilena (García et al., 2018) se encontró la misma estructura por medio de un análisis factorial confirmatorio. En este estudio se obtuvo una consistencia interna de sus subescalas que fluctuó entre $\alpha=.29$ —n autodistraccióny $\alpha=.95$ —n uso de sustancias-.

\section{Escala de satisfacción con la vida (SWLS)}

Se utilizó la versión validada por Arias y García (2018) de la Escala de satisfacción con la vida originalmente elaborada por Diener (1984), que consta de cinco ítems con alternativas de respuesta tipo Likert que oscilan entre 1 ("muy en desacuerdo") y 7 ("muy de acuerdo"). En este estudio se obtuvo una consistencia interna de $\alpha=.77$.

\section{Escala de estrés percibido (PSS-14)}

Esta escala, creada por Cohen et al. (1983), y adaptada para su uso en población chilena por Marín(2004), se utiliza para medir el grado en que los individuos evalúan situaciones de su vida como estresantes, y consta de 14 ítems que se contestan por medio de un formato tipo Likert con cinco opciones de respuesta —desde 0 ("nunca") hasta 4 ("muy a menudo")- En este estudio se obtuvo una consistencia interna de $\alpha$ de Cronbach $=.80$ para el total de la prueba.

\section{Severidad del dolor}

La severidad del dolor se evaluó por medio de dos preguntas. La primera, basada en la Escala numérica (Downie et al., 1978) — la más utilizada en el campo del dolor crónico- evalúa la intensidad del último episodio de cefalea en una escala de 1 ("nada intenso") a 10 ("muy intenso"); y la segunda, basada en la Escala de gradación del dolor — adaptada al español por Ferrer et al. (2016)-, evalúa la frecuencia de los episodios de cefalea en una escala que va de 1 ("una vez a la semana o menos") a 4 (“cuatro veces a la semana o más").

\section{Cuestionario sociodemográfico}

Se elaboró un cuestionario ad hoc en el que se registró información acerca del sexo, escolaridad, ocupación, estado civil, número de hijos, tipo de cefalea, y el sistema de salud por el cual estaban siendo tratados por su cefalea -público o privado-.

\section{Procedimiento}

Se realizó un muestreo por conveniencia para acceder a participantes con cefalea primaria, para lo cual se utilizó la técnica "bola de nieve", donde se comienza con pacientes conocidos del equipo investigador y luego con conocidos de estos pacientes. Los participantes fueron encuestados en forma presencial por dos de las investigadoras, y, previo a la aplicación de la encuesta, se solicitó a los participantes firmar un consentimiento informado en el que se aseguraba el carácter voluntario, anónimo y confidencial de los datos entregados.

\section{Aspectos éticos}

El estudio fue aprobado en sus aspectos éticos por una comisión de la Universidad de las Américas, Concepción, Chile, que evaluó el proyecto y el consentimiento informado.

\section{Análisis de datos}

Se utilizó el software estadístico SPSs 20 para el análisis de los datos. En una primera etapa, se realizó el análisis de distribución normal de las variables por medio de la prueba de Kolmogorov-Smirnov, donde se encontró una distribución normal; posteriormente, se realizó un análisis descriptivo de la muestra y de los instrumentos utilizados; a continuación, se correlacionaron las variables de estudio por medio del coeficiente de correlación de Pearson ( $r$ ), considerando para su interpretación que existe asociación entre las variables cuando, además de resultar significativas, las diferencias son superiores a .5 (Cohen, 1988), y en psicología superiores a .4 (Aron \& Aron, 2001) —para establecer las asociaciones de bienestar subjetivo y de estrés con las estrategias de afrontamiento, frecuencia e intensidad del dolor, se realizaron dos regresiones lineales múltiples-; $\mathrm{y}$, finalmente, se comparó lo reportado por las personas atendidas en el sistema de salud público con los datos de las que eran atendidas en el sistema particular por medio de la prueba $t$ de Student para muestras independientes.

\section{Resultados}

En primer lugar, en la Tabla 2 se puede encontrar el análisis descriptivo de las variables de estudio, en donde se observa que las estrategias de afrontamiento de afrontamiento activo, planificación y aceptación obtuvieron las medias más altas, mientras que el uso de sustancias obtuvo la media más baja. Además, en general, la consistencia interna de las escalas fue en su mayoría adecuada, excepto 
en las de algunas estrategias de afrontamiento, como la autodistracción, la aceptación y la desconexión, entre otras.

Tabla 2.

\begin{tabular}{lcccc} 
Estadísticos descriptivos de las variables de estudio $(n=150)$ \\
\hline & Mín. & Máx. & Media & $D E$ \\
\hline Bienestar subjetivo & 9 & 34 & 25.50 & 4.70 \\
Estrés percibido & 8 & 51 & 27.54 & 6.79 \\
EA - Afrontamiento activo & 0 & 6 & 3.85 & 1.42 \\
EA - Planificación & 0 & 6 & 3.49 & 1.45 \\
EA - Apoyo emocional & 0 & 6 & 2.62 & 1.53 \\
EA - Apoyo social & 0 & 6 & 2.40 & 1.44 \\
EA - Religión & 0 & 6 & 3.28 & 1.98 \\
EA - Reevaluación positiva & 0 & 6 & 3.11 & 1.30 \\
EA - Aceptación & 0 & 6 & 3.39 & 1.31 \\
EA - Negación & 0 & 6 & 1.31 & 1.36 \\
EA - Humor & 0 & 6 & 2.08 & 1.64 \\
EA - Autodistracción & 0 & 6 & 3.31 & 1.38 \\
EA - Autoinculpación & 0 & 6 & 2.15 & 1.56 \\
EA - Desconexión & 0 & 5 & 1.42 & 1.28 \\
EA - Desahogo & 0 & 6 & 2.02 & 1.38 \\
EA - Uso de sustancias & 0 & 6 & 0.64 & 1.30 \\
Intensidad del dolor & 2 & 10 & 6.72 & 1.81 \\
Frecuencia del dolor & 1 & 4 & 2.18 & 1.00 \\
\hline
\end{tabular}

Nota. EA = estrategia de afrontamiento.

Por otra parte, respecto a la relación entre las variables - como se puede observar en la Tabla 3-, destaca la correlación obtenida entre el bienestar subjetivo y el estrés percibido, que fue negativa $(r=-.30)$; así como que entre las estrategias de afrontamiento que correlacionan más alto con el bienestar subjetivo se encuentran la planificación $(r=.29)$ y el afrontamiento activo $(r=.28)$, mientras que con la frecuencia del dolor el bienestar subjetivo presentó una correlación inversa $(r=-.28)$. Asimismo, el estrés percibido presenta una mayor correlación con la negación $(r=.42)$, y la autoinculpación $(r=.31)$, mientras que con la aceptación presenta una correlación inversa $(r=-.33)$.

Si bien todas las correlaciones señaladas son significativas, de acuerdo con los criterios establecidos en el presente estudio, la única correlación de relevancia es la que ocurre entre el estrés percibido y la negación.

Posteriormente, se calcularon los predictores de bienestar subjetivo y estrés percibido por medio de la regresión lineal múltiple con el método de pasos sucesivos, el cual permite eliminar aquellas variables que no resultan significativas a la hora de predecir la variable dependiente. Así, en cuanto al bienestar subjetivo, el análisis muestra resultados significativos $\left(F_{(3,145)}=9.439 ; p<.001\right)$ con un $R^{2}$ de $.16\left(R^{2} a d j=.15\right)$, donde la planificación fue un predictor significativo positivo, mientras que la frecuencia del dolor y el uso de sustancias fueron predictores significativos negativos (véase Tabla 4).

En cuanto al estrés percibido, la regresión lineal múltiple mostró resultados significativos $\left(F_{(4,143)}=14.718 ; p<.000\right)$, con un $R^{2}$ de $.29\left(R^{2} a d j=.27\right)$, donde la frecuencia del dolor y las estrategias de afrontamiento de negación y autoinculpación fueron predictores significativos positivos, mientras que la aceptación resultó ser un predictor significativo negativo (véase Tabla 5).

De igual forma, se efectuó una comparación por sexo a través de la prueba $t$ de Student para muestras independientes,

Tabla 3.

Correlaciones de Pearson entre las variables de estudio

\begin{tabular}{lcc}
\hline \multicolumn{1}{c}{ Variable } & Bienestar subjetivo & Estrés percibido \\
\hline 1. EA - Afrontamiento activo & $0.28^{* * *}$ & -0.05 \\
2. EA - Planificación & $0.29^{* * *}$ & -0.01 \\
3. EA - Apoyo emocional & $0.17^{*}$ & 0.12 \\
4. EA - Apoyo social & 0.09 & $0.30^{* * *}$ \\
5. EA - Religión & 0.06 & 0.14 \\
6. EA - Reevaluación positiva & $0.17^{*}$ & $-0.19^{*}$ \\
7. EA - Aceptación & 0.14 & $-0.33^{* * *}$ \\
8. EA - Negación & -0.01 & $0.42^{* * *}$ \\
9. EA - Humor & 0.12 & -0.08 \\
10. EA - Autodistracción & $0.24 * *$ & -0.02 \\
11. EA - Autoinculpación & -0.02 & $0.31^{* * *}$ \\
12. EA - Desconexión & -0.02 & 0.14 \\
13. EA - Desahogo & 0.03 & 0.13 \\
14. EA - Uso de sustancias & -0.15 & $0.19^{*}$ \\
15. Frecuencia del dolor & $-0.28^{* * *}$ & $0.23^{* *}$ \\
16. Intensidad del dolor & 0.00 & 0.07 \\
\hline
\end{tabular}

Nota. $\mathrm{EA}=$ estrategia de afrontamiento. ${ }^{*} p<.05 ;{ }^{* *} p<.01 ;{ }^{* * *} p<.001$. 
Tabla 4.

Coeficientes y nivel de significación para los resultados del modelo de regresión lineal múltiple sobre el bienestar subjetivo en personas con cefalea primaria $(n=150)$

\begin{tabular}{|c|c|c|c|c|c|}
\hline & Coeficien & darizados & \multirow{2}{*}{$\begin{array}{c}\begin{array}{c}\text { Coeficientes } \\
\text { tipificados }\end{array} \\
\beta\end{array}$} & \multirow{2}{*}{ Valor $t$} & \multirow{2}{*}{ Valor $p$} \\
\hline & B & $\mathrm{EE}$ & & & \\
\hline (Constante) & 25.42 & 1.34 & & 19.029 & $<0.001$ \\
\hline Frecuencia del dolor & -1.13 & 0.37 & -0.24 & -3.083 & 0.002 \\
\hline EA - Planificación & 0.83 & 0.25 & 0.25 & 3.286 & 0.001 \\
\hline EA - Uso de sustancias & -0.56 & 0.28 & -0.15 & -2.016 & 0.046 \\
\hline
\end{tabular}

Nota. EA = Estrategia de afrontamiento.

Tabla 5.

Coeficientes y nivel de significación para los resultados del modelo de regresión lineal múltiple sobre el estrés percibido en personas con cefalea primaria

\begin{tabular}{|c|c|c|c|c|c|}
\hline & \multicolumn{2}{|c|}{ Coeficientes no estandarizados } & \multirow{2}{*}{$\begin{array}{c}\text { Coeficientes } \\
\text { tipificados } \\
\beta \\
\end{array}$} & \multirow[t]{2}{*}{ Valor $t$} & \multirow[t]{2}{*}{ Valor $p$} \\
\hline & B & $\mathrm{EE}$ & & & \\
\hline (Constante) & 24.64 & 2.17 & & 11.379 & $<0.001$ \\
\hline Frecuencia del dolor & 1.08 & 0.49 & 0.16 & 2.194 & 0.030 \\
\hline EA - Negación & 1.48 & 0.40 & 0.29 & 3.672 & $<0.001$ \\
\hline EA - Autoinculpación & 0.95 & 0.32 & 0.22 & 2.973 & 0.003 \\
\hline EA - Aceptación & -0.99 & 0.41 & -0.19 & -2.446 & 0.016 \\
\hline
\end{tabular}

Nota. EA = Estrategia de afrontamiento.

pero en los análisis solo se encontraron diferencias significativas en la frecuencia del dolor $\left(t_{(148)}=2.413, p=.017\right)$, donde las mujeres $(M=2.24 ; D E=1.01)$ reportaron una frecuencia más alta que los hombres $(M=1.60 ; D E=0.63)$.

Finalmente, mediante la $t$ de Student, se calcularon las diferencias de acuerdo con el sistema de salud en el cual los usuarios reciben atención — particular o público—, donde se encontraron diferencias significativas solo en el estrés percibido $\left(t_{(50)}=4.100, p<.001\right)$, pues las personas que asisten a atención pública presentaron más estrés $(M=31.54$; $D E=6.37)$ que quienes son atendidas en forma privada $(M=24.46 ; D E=6.06)$.

\section{Discusión}

El propósito de este estudio fue identificar los predictores de bienestar subjetivo y estrés percibido en personas afectadas por una cefalea primaria, y para ello se establecieron como variables predictoras distintas estrategias de afrontamiento, así como la intensidad y frecuencia del dolor percibido.

En general, se encontró una correlación negativa entre el estrés percibido y el bienestar psicológico, lo que reafirma lo encontrado en estudios previos (García et al., 2018), y que es comprensible por la asociación existente entre el estrés percibido y los distintos indicadores de malestar, como los síntomas depresivos y ansiosos (Barra et al., 2006; Espinosa et al., 2015).

Asimismo, al poner la atención sobre los predictores del bienestar, encontramos que la planificación influye positivamente, mientras que el uso de sustancias y la frecuencia del dolor influyen en forma negativa. Al respecto, estudios similares han mostrado que un afrontamiento centrado en la resolución de problemas - como lo es la planificaciónactúa como predictor de bienestar subjetivo (Eskin et al., 2014; García et al., 2016; Paris \& Omar, 2009), y que el uso de la planificación — como una estrategia de afrontamiento centrada en el problema - implica que la persona es capaz de actuar activamente para enfrentar y lidiar con los eventos estresantes y sus consecuencias, y, por lo tanto, implica una mayor percepción de control y autoeficacia (Chesney et al., 2006). De hecho, la relación entre autoeficacia y bienestar psicológico se ha encontrado en varios estudios (p. ej. Melato et al., 2017), y, por tanto, se podría sostener que la planificación posibilita el bienestar en las personas con cefalea, pues ayuda a enfrentar las diversas demandas gracias a que permite ordenar y programar acciones que promueven posibles soluciones.

Adicionalmente, el uso de sustancias aparece en nuestro estudio como un predictor negativo del bienestar, lo que reafirma lo encontrado en otros estudios (García et al., 2018). Respecto a esto, es probable que el ineficiente manejo del 
dolor por parte de los pacientes lleve a un aumento en la ingesta de distintos medicamentos, legales o no, destinados a paliar este dolor (Chowdhury, 2012), lo que hace que, contrario a la planificación, al tener que recurrir a medios externos para reducir el dolor, el uso de sustancias implique una menor percepción de control y de autoeficacia, lo que tendría como resultado una afectación negativa sobre la percepción de bienestar.

Por otra parte, al evaluar las variables que predicen estrés percibido, observamos que la negación y la autoinculpación aparecen como predictores positivos, mientras que la aceptación sería un predictor negativo. Con esto, se puede afirmar que negar la realidad del acontecimiento estresante y culpabilizarse por la situación ocurrida parecen potenciar el estrés - lo que coincide con estudios anteriores (p. ej., García et al., 2018)—, razón por la cual se les ha considerado tipos de afrontamiento desadaptativo y evitativo (Doron et al., 2014; Meyer, 2001); mientras que, por el contrario, aceptar la realidad del problema de salud disminuye los niveles de estrés, lo que podría deberse a que la aceptación del dolor tiende a reducir su intensidad, pues orienta a la persona a utilizar estrategias más efectivas para lidiar con el dolor (Kratz et al., 2007).

$\mathrm{Al}$ respecto, García et al. (2016) han señalado que la aceptación de que una enfermedad está fuera de su control lleva a las personas a resignarse ante aquello que no se puede cambiar, lo que permite concentrar sus esfuerzos en aquellos aspectos que sí están bajo su control, como la planificación de acciones de cuidado y autocuidado.

Otro hallazgo importante es que la frecuencia de episodios de cefaleas predice tanto la disminución del bienestar como el aumento del malestar, incluso más que la intensidad del dolor. Este resultado coincide con otras investigaciones (p. ej., Schramm et al., 2014), donde se ha encontrado que cuando la frecuencia del dolor aumenta a tal punto de volverse crónico, las personas tienden a reportar una disminución del bienestar, debido a las limitaciones que produce, al deterioro en la calidad de vida (Díaz \& Marulanda, 2011), así como a la disminución en la capacidad de desarrollar las actividades cotidianas y la pérdida de la competencia laboral (Domínguez, 2007).

Ahora bien, la frecuencia del dolor también marcó una diferencia significativa entre mujeres y hombres, pues se encontró una frecuencia más alta en las primeras. Esto coincide con el estudio de Guic et al. (2002), en el cual se mostró que las mujeres presentaron mayor percepción de molestias corporales - como dolores musculares en el cuello, cara y brazos, entre otros-que los hombres. De hecho, la oms (2006) ha señalado que son las mujeres las que experimentan con mayor frecuencia cefaleas primarias.

Finalmente, al comparar el sistema de salud por el cual están siendo tratadas las personas con cefalea primaria, se evidenció que quienes son atendidas en el sistema público presentan mayores niveles de estrés que quienes asisten a consultas particulares. Esto se puede entender por las enormes diferencias en la calidad de la atención entre los servicios públicos y privados, pues los primeros presentan mayores tiempos de espera, así como menor tiempo de interacción con el profesional, entre otros factores (Riveros \& Berné, 2007; Silva, 2018) que pueden asociarse a mayores estresores y menores posibilidades de que los usuarios del sistema público puedan realizar actividades de distracción y relajación al momento de ser atendidos.

Ahora bien, es importante plantear algunas limitaciones del presente estudio, como que su diseño fue no experimental y transversal, lo que impide establecer relaciones causa y efecto; que los participantes fueron elegidos por accesibilidad, y por lo tanto no necesariamente representan a la población general que sufre de problemas de cefalea primaria; y que algunas subescalas del Brief Cope presentaron consistencias internas inadecuadas, lo que ha sido una dificultad común en este instrumento, explicable por el hecho de que cada subescala está constituida por solo dos ítems, un número inadecuado para evaluar tanto la confiabilidad (García et al., 2018) como la validez de la prueba (Lloret et al., 2014).

No obstante, se puede concluir que el presente estudio se dirigió específicamente a identificar las estrategias de afrontamiento que contribuyen a un mejor manejo del estrés en personas con cefalea primaria y que, por tal razón, permiten tanto un mayor bienestar subjetivo como la reducción del estrés. De este modo, esperamos que los resultados obtenidos sean de utilidad en la práctica del ejercicio clínico de los profesionales de la salud al momento de atender a pacientes con este tipo de características, y que permitan generar una reflexión sobre el tipo de atención que se ofrece en el sistema público. En concreto, se podría establecer que incentivar el uso de la planificación para afrontar el malestar o sus consecuencias, además de promover la aceptación de la enfermedad, contribuirían positivamente en la salud mental; mientras que el uso frecuente de sustancias, las estrategias evitativas y la frecuencia del dolor influyen negativamente, $\mathrm{y}$, por tanto, deterioran su percepción de bienestar. Creemos que con estos hallazgos podremos contribuir a mejorar la calidad de vida de las personas que padecen de algún tipo de cefalea primaria. 


\section{Referencias}

Arias, P. R., \& García, F. E. (2018). Propiedades psicométricas de la Escala de Satisfacción con la Vida en población ecuatoriana adulta. Pensamiento Psicológico, 16(2), 21-29. https://doi.org/10.11144/Javerianacali.PPSI16-2.ppes

Aron, A., \& Aron, E. N. (2001). Estadística para psicología (2. ${ }^{\mathrm{a}}$ ed.). Pearson.

Barra, E., Cerna, R., Kramm, D., \& Véliz, V. (2006). Problemas de salud, estrés, afrontamiento, depresión y apoyo social en adolescentes. Terapia Psicológica, 24(1), 55-61. https:// www.redalyc.org/pdf/785/78524106.pdf

Benito-González, E., Palacios-Ceña, M., Fernández-Muñoz, J. J., Castaldo, M., Wang, K., Catena, A., Arendt-Nielsen, L., \& Fernández, C. (2018). Variables associated with sleep quality in chronic tension-type headache: A cross-sectional and longitudinal design. PloS One, 13(5), e0197381. https:// doi.org/10.1371/journal.pone.0197381

Carver, C. (1997). You want to measure coping but your protocol's too long: Consider the Brief COPE. International Journal of Behavioral Medicine, 4(1), 92-100. https://doi. org/10.1207/s15327558ijbm0401_6

Carver, C. S., Scheier, M. F., \& Weintraub, J. K. (1989). Assessing coping strategies: A theoretically based approach. Journal of Personality and Social Psychology, 56, 267-283. https://doi.org/10.1037/0022-3514.56.2.267

Chesney, M. A., Neilands, T. B., Chambers, D. B., Taylor, J. M., \& Folkman, S. (2006). A validity and reliability study of the Coping Self-efficacy Scale. British Journal of Health Psychology, 11(3), 421-437. https://doi. org/10.1348/135910705X53155

Chowdhury, D. (2012). Tension type headache. Annals of Indian Academy of Neurology, 15(1), 83-88. https://doi. org/10.4103/0972-2327.100023

Cohen, J. (1988). Statistical power analysis for the behavioral sciences. Erlbaum.

Cohen, S., Kamarck, T., \& Mermelstein, R. (1983). A Global Measure of Perceived Stress. Journal of Health Social Behavior, 24(4), 385-396. https://doi.org/10.2307/2136404

Cohen, M., Quintner, J., \& van Rysewyk, S. (2018). Reconsidering the International Association for the Study of Pain definition of pain. Pain Reports, 3(2), e643. https:// doi.org/10.1097/PR9.0000000000000643

Del Monaco, R. (2013). Dolor crónico y narrativa: Experiencias cotidianas y trayectorias de atención en el padecimiento de la migraña. Physis: Revista de Saúde Coletiva, 23(2), 489510. https://doi.org/10.1590/s0103-73312013000200009

Díaz, R., \& Marulanda, F. (2011). Dolor crónico nociceptivo y neuropático en población adulta de Manizales (Colombia). Acta Médica Colombiana, 36(1), 10-17. http://www.scielo. org.co/pdf/amc/v36n1/v36n1a03.pdf

Diener, E. (1984). Subjective well-being. PsychologicalBulletin, 95, 542-575. https://doi.org/10.1037/0033-2909.95.3.542
Diener, E., Oishi, S., \& Lucas, R. E. (2003). Personality, Culture, and Subjective Well-Being: Emotional and Cognitive Evaluations of Life. Annual Review of Psychology, 54(1), 403-425. https://doi.org/10.1146/an nurev.psych.54.101601.145056

Domínguez, B. (2007). Analgesia hipnótica en el dolor crónico. Revista Iberoamericana del Dolor, 2(4), 24-30. https://doc player.es/84139312-Revista-iberoamericana-del-dolor.html

Doron, J., Trouillet, R., Gana, K., Boiché, J., Neveu, D., \& Ninot, G. (2014). Examination of the hierarchical structure of the Brief COPE in a French sample: Empirical and theoretical convergences. Journal of Personality Assessment, 96(5), 567-575. https://doi.org/10.1080/00223891.2014.88 6255

Downie, W. W., Leatham, P. A., Rhind, V. M., Wright, V., Branco, J. A., \& Anderson, J. A. (1978). Studies with pain rating scales. Annals of the Rheumatic Diseases, 37(4), 378381. https://doi.org/10.1136/ard.37.4.378

Dowson, A. J. (2003). Analysis of the patients attending a specialist UK headache clinic over a 3-year period. Headache: The Journal of Head and Face Pain, 43(1), 14-18. https:// doi.org/10.1046/j.1526-4610.2003.03003.x

Eskin, M., Şavk, E., Uslu, M., \& Küçükaydoğan, N. (2014). Social problem-solving, perceived stress, negative life events, depression and life satisfaction in psoriasis. Journal of the European Academy of Dermatology and Venereology, 28(11), 1553-1559. https://doi.org/10.1111/jdv.12355

Espinosa, M. C., Orozco, L. A., \& Ybarra, J. L. (2015). Síntomas de ansiedad, depresión y factores psicosociales en hombres que solicitan atención de salud en el primer nivel. Salud Mental, 38(3), 201-208. https://doi.org/10.17711/ sm.0185-3325.2015.028

Ferrer, R., Gil, A., Pardo, J., Jiménez, V., Gallego, T., \& La Touche, R. (2016). Adaptación y validación de la Escala de Gradación del Dolor Crónico al español. Reumatología Clínica, 12(3), 130-138. https://doi.org/10.1016/j. reuma.2015.07.004

Fumal, A., \& Schoenen, J. (2008). Tension-type headache: Current research and clinical management. The Lancet Neurology, 7(1), 70-83. https://doi.org/10.1016/ S1474-4422(07)70325-3

Garcia, F., Barraza, C. G., Wlodarczyk, A., Alveal, M., \& Reyes, A (2018). Psychometric properties of the Brief-COPE for the evaluation of coping strategies in the Chilean population. Psicologia: Reflexão e Crítica, 31(22), 1-11. https:// doi.org/10.1186/s41155-018-0102-3

García, F., Manquián, E., \& Rivas, G. (2016). Bienestar psicológico, estrategias de afrontamiento y apoyo social en cuidadores informales. Psicoperspectivas, 15(3), 87-97. https://doi. org/10.5027/psicoperspectivas-vol15-issue3-fulltext-770

Girdano, D., \& Everly, G. S. (1986). Controlling Stress and Tension (2. ${ }^{\mathrm{a}}$ ed.). Prentice-Hall.

Guic, E., Bilbao, M., \& Bertin, C. (2002). Estrés laboral y salud en una muestra de ejecutivos chilenos. Revista Médica 
de Chile, 130(10), 1101-1112. https://doi.org/10.4067/ S0034-98872002001000004

Hernández, R., Fernández, C., \& Baptista, P. (2003). Metodología de la Investigación. Mc Graw Hill.

Hofstra, W. A., Hageman, G., \& de Weerd, A. W. (2015). Headache in epilepsy patients: The (un) awareness of this phenomenon among Dutch neurologists. Seizure, 25, 37-39. https://doi.org/10.1016/j.seizure.2014.12.004

International Association for the Study of Pain (1979). Pain terms: a list with definitions and notes on usage. Recommended by the IASP Subcommittee on Taxonomy. Pain, 6(3), 249-252. https://pubmed.ncbi.nlm.nih. gov/460932/

International Headache Society (2018). The International Classification of Headache Disorders. Cephalalgia, 38(1), 1-211. https://doi.org/10.1177/0333102417738202

Kratz, A. L., Davis, M. C., \& Zautra, A. J. (2007). Pain acceptance moderates the relation between pain and negative affect in female osteoarthritis and fibromyalgia patients. Annals of Behavioral Medicine, 33(3), 291-301. https://doi. org/10.1007/BF02879911

Lazarus, R., \& Folkman, S. (1984). Stress: Appraisal and coping. Springer Publishing Company.

Lloret, S., Ferreres, A., Hernández, A., \& Tomás, I. (2014). El análisis factorial exploratorio de los ítems: una guía práctica, revisada y actualizada. Anales de Psicología, 30(3), 1151-1169. http://scielo.isciii.es/pdf/ap/v30n3/metodologia 1.pdf

Marín, E. (2004). Estandarización de las escalas, Perceived Stress Scale de Cohen (PSS-14), Escala Magallanes de estrés (Emest), y estudio comparativo de ambas escalas en una muestra de profesionales del área metropolitana de Santiago de Chile (Tesis de grado). Universidad Santo Tomás, Santiago de Chile.

Martin, P. R. (2016). Stress and primary headache: Review of the research and clinical management. Current Pain and Headache Reports, 20(7), 1-8. https://doi.org/10.1007/ s11916-016-0576-6

Melato, S. R., van Eeden, C., Rothmann, S., \& Bothma, E. (2017). Coping self-efficacy and psychosocial well-being of marginalised South African youth. Journal of Psychology in Africa, 27(4), 338-344. https://doi.org/10.1080/14330237.2 017.1347755

Meyer, B. (2001). Coping with severe mental illness: Relations of the Brief COPE with symptoms, functioning, and well-being. Journal of Psychopathology and Behavioral Assessment, 23(4), 265-277. https:/doi. org/10.1023/a:1012731520781

Ministerio de Salud, Chile (2006). Segunda encuesta de calidad de vida y salud chile 2006. Subsecretaria de Salud. http:// www.crececontigo.gob.cl/wp-content/uploads/2015/11/ ENCAVI-2006.pdf
Moretti, L., \& Medrano, L. (2014). Validación de cuestionario de conductas de dolor en estudiantes universitarios con cefaleas. Liberabit, 20(1), 101-107. http://www.scielo.org.pe/ pdf/liber/v20n1/a09v20n1.pdf

Najam, N., \& Aslam, S. (2010). Perceived stress and coping strategies in headache (migraine \& tension-type headache) patients. Journal of Behavioural Sciences, 20(1), 1-15. https://www.semanticscholar.org/paper/PerceivedStress-and-Coping-Strategies-in-Headache-Najam-Aslam/ d8cd811ad2a0226100fddc9a44603ef2bd5860f5

Operto, F. F., Craig, F., Peschechera, A., Mazza, R., Lecce, P. A., \& Margari, L. (2018). Parenting stress and emotional/ behavioural problems in adolescents with primary headache. Frontiers in Neurology, 8(749), 1-5. https://doi. org/10.3389/fneur.2017.00749

Organización Mundial de la Salud [OMS]. (2006). Trastornos Neurológicos desafios para la salud pública. OMS. https://www.paho.org/hq/dmdocuments/2008/Trastornos_ Neurologicos.pdf

Organización Mundial de la Salud [OMS]. (2011). Atlas of headache disorders and resources in the world 2011. World Health Organization. https://apps.who.int/iris/bitstream/ handle/10665/44571/9789241564212_eng.pdf

Organización Mundial de la Salud [OMS]. (2016). Cefaleas. World Health Organization. https://www.who.int/es/ news-room/fact-sheets/detail/headache-disorders

Organización Mundial de la Salud [OMS]. (2019). CIE-11 para estadisticas de mortalidad y morbilidad. World Health Organization. https://icd.who.int/browse11/1-m/es\#/http:// id.who.int/icd/entity/2104869000

Paris, L., \& Omar, A. (2009). Estrategia de afrontamiento de estrés como potenciadoras de bienestar. Psicología y Salud, 19(29), 167-175. https://doi.org/10.25009/pys.v19i2.626

Pascual, J. (2019). Cefalea y migraña. Medicine - Programa de Formación Médica Continuada Acreditado, 12(71), 41454153. https://doi.org/10.1016/j.med.2019.01.010

Ramírez, C. (2002). Características personales en la experiencia de dolor y en el proceso de afrontamiento. Escritos de Psicología, 6, 40-52. https://dialnet.unirioja.es/descarga/ articulo/281675.pdf

Riveros, J., \& Berné C. (2007). Análisis de la opinión de usuarios sobre calidad percibida y satisfacción con hospitales públicos: Estudio de caso desde la perspectiva de la aplicación del marketing. Revista Médica Chile, 135(7), 862-870. https://doi.org/10.4067/S0034-98872007000700006

Silva, L. (2018). Análisis de las desigualdades sociales en Chile entre los años 1992 y 2013. Oportunidades vitales, necesidades de los hogares y diferencias regionales (Tesis doctoral). Universidad de Barcelona, España.

Schramm, S. H., Moebus, S., Lehmann, N., Galli, U., Obermann, M., Bock, E., Yoon, M., Diener, H., \& Katsarava, Z. (2014). The association between stress and headache: A longitudinal 
population-based study. Cephalalgia, 35(10), 853-863. https://doi.org/10.1177/0333102414563087

Tomé-Pires, C., Solé, E., Racine, M., Galán, S., Castarlenas, E., Jensen, M. P., \& Miró, J. (2016). The relative importance of anxiety and depression in pain impact in individuals with migraine headaches. Scandinavian Journal of Pain, 13(1), 109-113. https://doi.org/10.1016/j.sjpain.2016.08.002

Torres, W., \& Flores, M. (2018). Factores predictores del bienestar subjetivo en adultos mayores. Revista de Psicología (PUCP), 36(1), 9-48. https://doi.org/10.18800/ psico.201801.001

Vos, T., Allen, C., Arora, M., Barber, R. M., Bhutta, Z. A., Brown, A., ... Coggeshall, M. (2016). Global, regional, and national incidence, prevalence, and years lived with disability for 310 diseases and injuries, 1990-2015: A systematic analysis for the Global Burden of Disease Study 2015. The Lancet, 388(10053), 1545-1602. https://doi. org/10.1016/S0140-6736(16)31678-6

Waldie, K., Buckley, J., Bull, P. N., \& Poulton, R. (2015). Tension-type headache: A life-course review. Journal of Headache \& Pain Management, 1(1-2), 1-9. https://doi. org/10.4172/2472-1913.100002 